This is a self-archived version of an original article. This version may differ from the original in pagination and typographic details.

Author(s): Garda, Maria B.; Karhulahti, Veli-Matti

Title: Let's Play Tinder! : Aesthetics of a Dating App

Year: 2021

Version: Accepted version (Final draft)

Copyright: @ 2019, SAGE Publications

Rights: In Copyright

Rights url: http://rightsstatements.org/page//nC/1.0/?language=en

Please cite the original version:

Garda, M. B., \& Karhulahti, V.-M. (2021). Let's Play Tinder! : Aesthetics of a Dating App. Games and Culture : A Journal of Interactive Media, 16(2), 248-261.

https://doi.org/10.1177/1555412019891328 
Preprint.

Full reference: Garda, M. B., \& Karhulahti, V. M. (2019). Let's Play Tinder! Aesthetics of a Dating

App. Games and Culture, 1555412019891328. DOI: 10.1177/1555412019891328

\title{
Let's Play Tinder! Aesthetics of a Dating App
}

\begin{abstract}
This article provides an analysis of the "dating app" Tinder as an aesthetic ludic artifact. By scrutinizing the title's features of gameplay and expressive-interpretive social interaction, Tinder usage is set into a frame theory context and shown to operate by multiple overlapping frames that allow romantic engagement to be entered as play and vice versa.
\end{abstract}

Keywords

aesthetics, Tinder, dating app, gameplay, social media 


\author{
Preprint. \\ Full reference: Garda, M. B., \& Karhulahti, V. M. (2019). Let's Play Tinder! Aesthetics of a Dating \\ App. Games and Culture, 1555412019891328. DOI: 10.1177/1555412019891328
}

\title{
Introduction
}

Tinder, a mobile "dating app," has changed human practices of forming relation- ships to a degree that shakes both mainstream journalism and software sales (e.g., Fox, 2016; Paul, 2018; Summers, 2014). This article provides an analysis of the app's aesthetic user experience as a form of play and how this reflects present-day ludification processes.

We take off from multiple research findings which state that the primary motiva- tion for Tinder use is not to pursue a dating partner (unlike its label implies) but rather to employ the software as an entertainment medium (e.g., Carpenter \& McE- wan, 2016; Sumter, Vandenbosch, \& Ligtenberg, 2017; Timmermans \& De Caluwe', 2017b; see also Gatter \& Hodkinson, 2016; Sevi, Aral, \& Eskenazi, 2018). In a comprehensive analysis of Tinder's networked user functions, Duguay (2017) elaborates on such findings by showing how the app "sustains enrolment through game-like activity flows [where] repetitive and fast-paced swiping is designed to invoke ongoing participation" (p. 360). With reference to a number of other sources, she further notes that "Tinder's new algorithm ranks users according to how suc- cessfully they match [which] provides incentive to strategically play the game, generating reputation-based authenticity" (pp. 360).

While Tinder's ludic identity and interface have thus already been entertained by academics in previous literature (see also Derk, 2016; Ward, 2017), the techniques by which Tinder constructs a distinct interpretive-kinesthetic play experience have not gained scholarly attention yet. Hence, through the methodological means of feature analysis (not interface analysis), we provide an examination of Tinder software and how it frames an aesthetic ludic experience as a form of play.

The first section uncovers the mechanics of Tinder gameplay. The second section probes the expressive and interpretive limits that delineate how users play the app. The third section exerts an examination of Tinder play via frame theory and shows how the app fuels a cycle of overlapping frames that maintain a process of constant reframing (and replaying).

\section{Gameplay}

"Gameplay" is one of the most controversial (but also one of the most central) concepts in the ongoing scholarly discourse on play and games. While the term's historical role in popular journalism served primarily to aid the gaming public in constructing a specific social identity in the 1980s and 1990s (Kirkpatrick, 2015), contemporary academics tend to employ it for delineating the experiential qualities of play activities (e.g., Ermi \& Mäyrä, 2005; Perron \& Arsenault, 2008; Vahlo, 2018). In the present study, we consult the latter: By Tinder's gameplay, we refer to the subjective, socially conscious phenomenological whole that delineates the app's utility as a ludic product. The below feature analysis is based on both Android and iPhone versions of the Tinder software which were active between 2017 and 2018.

According to its developer, Tinder is an "app for meeting new people" and to "spark something new" (Tinder.com). Regardless of what the words "meeting" and "something" mean for the individual, a compulsory requirement for each player is to run through an individualized avatar generation process: establish a profile with one's age, gender, and an uploaded image. Additionally, one may upload up to five extra images, write a description within 500 characters, assign school or work affiliation, choose a favorite song, and connect the profile to an Instagram account. The software is location based, which means that every user also shares their loca- tion in real time, all the time. Unlike in many video games, the above data should correspond with the player's actual personal profile; however, using tweaked or other data is a common strategy (more about this later).

In line with the app's official stated function of facilitating connections between people, the player also needs to set a target group (alterable afterward) that deter- mines who they are potentially matched with. The 
group is modified by three factors: gender (female/male/both), age (+10 years by default), and real-time geographical distance (up to a 100 miles from the present location). From here, players begin their quest for "matches."

Matching with another person on Tinder surfaces through a simple mechan- ical procedure. This procedure is embedded in "swiping," which can be consid- ered the app's "core mechanic," as it is the central action that players perform (most) repeatedly among other actions (see Järvinen, 2008; Karhulahti, 2011). Weiser et al., (2018) description handily sums up the desired effect of this swiping:

Tinder uses the GPS feature of the user's mobile phone to show nearby eligible partners' profiles. When users are interested in someone, they anonymously swipe to the right on their phone; when they are not interested in someone, they swipe to the left. When two people swipe right for each other, they are considered matched and are now able to communicate with one another. (p. 29)

While the haptic element in swiping left or right is fundamental to Tinder's repetitive gameplay, it is important not to overlook the range of cognitive processes behind "being interested or not," which is but one of the reasons for players to accept and reject profiles. As in all other kinds of structured play, Tinder provides its players with a multitude of goals to choose from; accordingly, previous studies have discovered Tinder players being motived by a need to feel that they belong, a desire to contact locals, curiosity satisfaction, friendly communication, love, passing of time, peer-pressure gratification, self-worth validation, sex, and the corroboration of their own trendiness (Ranzini \& Lutz, 2017; Sumter et al., 2017; Timmermans \& De Caluwe', 2017a).

While the above list is hardly comprehensive, it shows that the player's choice between right ("like") and left ("nope") swiping is hardly a simple matter of being "interested" in the person or not. For instance, players who are motivated primarily by self-worth validation or passing of time may well swipe right (or left) even though they are not interested in the target profile. With this factor in mind, the "like" and "nope" labels that define the core mechanic are first and foremost sym- bolic and (not unlike "running," "shooting," and "driving" in video games) mainly signify a virtual act that has predetermined software-specific consequences in the ludic system. The action of "shooting" in a video game does not involve discharging an actual projectile, but it is a symbolic framing of a specific in-game process that allows players, for instance, to accumulate points. In the same way, the player does not really "like" someone on Tinder by swiping their photo; the swipe is a mechanic that allows players to match with others and open direct communication channels with them.

In Tinder's in-game economics, matches function as the key currency. A match materializes when two players like each other, and as a consequential ludic premise, success in Tinder is generally defined by one's capacity to acquire (one or more) matches. Notably, matches respect a double-blind principle that is perhaps the most important of the app's technologically reinforced rules: Each player likes without knowing if the respective others like back. This means that players will never know if another player likes back unless both choose to like each other. These social dynamics constitute a case of mathematical game theory to which we return in the next section. At this point, it is worth emphasizing that due to the infinite (yet regulated) number of "likes" afforded to players and the absolute lack of obligations related to matching, for those interested in making more than one match (whatever the reason) liking a profile is somewhat logical.

Whether left or right, Tinder encourages users to swipe repeatedly. Again, the choice making processes that ultimately lead to left and right swipes differ between individuals and involve various strategic subactions. While the default action at facing a new profile (image) is to execute a binary swipe, ${ }^{1}$ the player can also choose to browse the profile's supplementary images, explore the (optional) textual descrip- tion, or enter (likewise optional) Instagram content. At the time of writing, these three extra actions (see more images, read text, and enter Instagram) demarcate the player's limited agency somewhat completely and, as such, provide a "possibility space" (Bogost, 2007), where certain modes of behavior appear more interesting (and rewarding) than others. 
According to the company's own statement, a single session of swiping takes about $7.2 \mathrm{~min}$ (for men) or 8.5 min (for women), and the average player commences a new session no less than 11 times per day (Bilton, 2014). David and Cambre (2016) aptly point out how Tinder's "simplification of choice by reducing it to a binary" (p. 7) efficiently encourages accelerated swiping sessions. Comparing the binary to alternative dating apps that additionally provide "maybe" and other options, the scholars argue for a specific "swipe logic" in which speed is intended by design. In concert with David and Cambre's swipe logic, Tinder's possibility space provides concerned players the chance for pausing (or slowing down) for some seconds before resuming to swipe; that is to say, the scarcity of browsable data (up to mere five more pictures, a text of 500 characters max, and social media connection) paces the minutes-long swipe flow to which players return some dozen times per day.

There are parallels to be drawn here between Tinder and slot machine gambling. In both Tinder and slot machine play, uninterrupted engagement is strongly related to the contingency factor of providing the individual with an endless chain of semirandom stimuli: A new profile image or hand of cards that requires small interpretive-kinesthetic input to be transformed into a matching set, thus maintaining a pleasurable state of uncertainty (cf. Costikyan, 2013). Compared to most other forms of gambling, slot machines enable their users to easily play a "match" every $3 \mathrm{~s}$, which can be considered, in behavioral psychology terms, an intensive instance of event frequency (see Schu"ll, 2012). While there is no citable research on the event frequencies of Tinder players (but see Banks, Westerman, \& Sharabi, 2017), the software facilitates a pace that produces one or more (potential) matches every few seconds. With reference to the numbers stated by its developer, it is unlikely that Tinder players ever lapse into the hours-long "zones" which addicted gamblers are known for (e.g., Livingstone, 2005); nevertheless, as reinforcing technological engagement, the core mechanical returns of Tinder swiping operate under a parallel principle.

\section{Expression and Interpretation}

Alongside gameplay, Tinder is a medium of communication that takes place in two respective virtual locations: statically presented (but ever modifiable) profile space and personal one-to-one text chat. Both locations operate under semiotic principles that vary based on multiple cultural, regional, and social factors. In pragmatic terms, for a player to attract like(s) from their potential coplayer(s), they need to construct a profile that appeals to the subject. Furthermore, if they wish to advance the formed relationship, interaction continues in a personal one-to-one text chat lobby that emerges as a reward for each successful match. Below, we look at both virtual locations and their semiotic principles.

Tinder players engage in environment-specific impression construction by decid- ing which images, descriptions, and other pieces of information to present. Opti- mally, the decision process serves their subjective goals and aids them in succeeding as (particular) players. Due to Tinder's design choices against antisocial behavior (profiles are supposed to represent actual individuals, hence being attached to registered devices and easily accessible reporting functions), those who wish to triumph tend to express themselves by accurate means, with the caveat that presenting oneself in an ideal (rather than realistic) light is part of the game. This strategic authenticity and impression management form the base of Tinder's gaming rules (see Sumter et al., 2017; Duguay, 2017; Tyson, Perta, Haddadi, \& Seto, 2016).

Keeping in mind that Tinder play operates largely by the core mechanic of repeated swiping, perhaps the most significant element of signification in the system is the initial profile image. Since many players make their negative and positive decisions along the flow of swiping, the first impression given by the initial profile image is decisive. Hence, the players' efforts to present themselves "likable" by means of visual self-image (in a way that appeals to their subjective goals) and to identify the self-images of potential matches (in concert with subjective goals) form an explicit system of expression and interpretation where details gain meaning as codes and cues. 
In a recent study by Qiu, Lu, Yang, Qu, and Zhu (2015), the group compared the selfies of 612 participants to their actual personality traits and the ways in which they were interpreted by others. The results indicated several cues reflecting the charac- teristics of the individuals' corresponding personality traits; for instance, "emotional positivity predicts agreeableness and openness, duckface indicates neuroticism, and private location in the background indicates less conscientiousness" ( $p$. 447). Those observing the selfies, however, could only accurately judge the selfie owners' degree of openness and used many invalid cues to judge their personality:

duckface was related to the judgment of conscientiousness and neuroticism, pressed lips were associated with the judgment of extraversion and openness, public location in the background was related to the judgment of conscientiousness, and being alone was related to neuroticism ratings. (p. 448)

While selfies naturally represent merely one form of self-presentation and Tinder images need not be selfies to begin with, the case illustrates how platform-specific imagery gets interpreted as personality-related cues or codes (consciously and not). By uploading selected profile pictures, players construct an exclusive (positive) image of themselves with hopes to convey that (positive) image by means of the app's visual system of cues and codes (cf. Hardey, 2008).

In addition to the signifiers of body (pose), clothing (style), and face (expression), profile images may include entities that describe the individual beyond their phys- ical persona. For instance, cars and other status items can be used to express finan- cial welfare, whereas books and other taste signifiers represent aesthetic preferences or cultural knowledge. Along similar lines, photos depicting food, pets, and travel indicate experiential varieties that thus become part of the individual's profile- mediated expression of self.

The same applies to the description box, which provides 500 characters of text to be used for verbal expression. While this space can be left empty, players are inclined to fill it with elaborating data about their motivations, inclinations, occu- pations, and personalities. The individual's manner of writing (or textual expression) is a factor that conveys and contributes to their play; for instance, irony and wittiness are frequently used to invite certain match types (see David \& Cambre, 2016). In place of the verbal, emojis operate in similar ways with their own semiotic depth: single-gendered human emojis routinely can be used to signal the individual having a daughter or son, whereas explicit drink, food, and sports emojis indicate leisure preferences.

While there is no guarantee that the information presented on players' profiles get interpreted in the intended way, the above patterns add to a platform-specific envi- ronment of expression and interpretation. Tinder players perceive the verbal-visual imagery via codes and cues that they have come to structure on the grounds of earlier play, thus constituting explicit ludo-hermeneutic circles (see Sicart 2011; Arjoranta, 2015; Karhulahti, 2015) supported by the game software.

\section{Frame Play}

For the present-day students of play and games, "magic circle" is a commonly used metaphor for explicating (and simplifying) ludic behavior. In principle, acts of play are said to constitute socially rigid spatiotemporal events in which things and actions gain unique meanings. For instance, a ball and the act of kicking have exclusive values in a soccer match, whereas the same ball and kicking action are somewhat differently valued in circus performances, museums, and so on. The unique "magic circle" of soccer enables distinguishing the game as soccer per se and, at the same time, circumscribing the purposes and policies concerning the things and actions within it (see Consalvo, 2009; Stenros, 2014; Paasonen 2018).

The magic metaphor is often incapable of explaining the complexities concerning diverse play and game activities, for which recent scholars have come to employ more advanced theoretical tools in their scrutinies of social ludic engagement. Perhaps the most known of these tools is the so-called frame analysis, as 


\section{Preprint. \\ Full reference: Garda, M. B., \& Karhulahti, V. M. (2019). Let's Play Tinder! Aesthetics of a Dating App. Games and Culture, 1555412019891328. DOI: 10.1177/1555412019891328}

restructured by Goffman (1974/1986). While earlier research on Tinder frequently mentions Goffman in passing (David \& Cambre, 2016; Duguay, 2017; Ranzini \& Lutz, 2017), at the time of writing, Ward (2017) is the only one with an explicit reference that dovetails into present analysis:

Goffman classically argues that individuals attempt to control or guide others' impres- sions by manipulating setting, appearance, and behavior [i.e.] people explicitly choose the impression they want to make and decide the method they will use to create it.... In Goffman's terms, Tinder provides the user a stage where s/he can perform. (p. 1646)

Again, Tinder is a digital environment that provides its performing players various subjectively selectable goals. This means that, not unlike in everyday inter- action, it can be difficult or impossible to know why a specific individual performs in a way they do, and the performing individual may not always be aware of their actual motivations either. Ultimately, then, social interaction in (and via) Tinder is a complicated net of actions and occurrences, the identity of which varies by the frame through which one looks at them. Goffman (1974/1986) elaborates:

characterization of the "same" event or social occasion may differ widely...it has been argued, for example, that opposing rooters at a football game do not experience the "same" game, and that what makes a party a good one for a participant who is made much of is just what makes it a bad one for a participant who thereby is made little of (pp. 9-10)

Along these lines, it is worth continuing with the premise that the "same" image or description in a Tinder profile may well be framed as an instrument for "discovering a lifelong partner," "luring hookups," "accumulating match col- lection," "meeting a new friend," or perhaps just "finding someone to chat with." This naturally applies to those who interpret and respond to such images and descrip- tions too; and of crucial note, none of the framings need to be the "actual" or "correct" one (cf. Bullingham \& Vasconcelos, 2013).

Looking at Tinder play from the frame-analytical viewpoint helps explain some of its socio-aesthetic idiosyncrasies. First and foremost, Tinder's status as a mobile app enables its players to reframe romantically and sexually associated actions into "fun" ludic engagement with "entertainment" technology, and this works the other way around too: Those who are playing in order to find the "right one" are also immersed in the same playful and ludically rewarding circuits of swiping as those with other motivations.

In a study published in Journal of Behavioral Addictions, Orosz, To'th-Kira'ly, B \}othe, and Melher (2016) present a Problematic Tinder Use Scale based on a factor analytic scrutiny of 430 Tinder players. As an incidental observation (with reference to external reports indicating almost half of Tinder users not being single), the scholars suggest that playing Tinder "can also be equally rewarding to those who are in stable relationships" (p. 521). Unsurprisingly, the suggestion was supported by their findings concerning problematic use according to which "no salient demography-related differences were found, including the relationship status" (p. 522). While it is possible that the numbers reflect Tinder's parallel utility as an infidelity tool (see Weiser et al., 2018; Timmermans, De Caluwe', \& Alexopoulos 2018), Orosz and others point about Tinder's relationship-neutral ludic rewards still make sense: By allowing one to frame the activity as "fun" and "entertainment" that is "nonserious" and free from romantic goals, the app constructs a ludic environment (and a game-like "magic circle") where emotionally and sexually exciting social interaction can (but need not) be "just a game."

Here, the previously mentioned qualitative work by Ward (2017) makes a point of empirical reflection. One of Ward's interviewees, Erwin, efficiently demonstrates how the framing of Tinder as a form of play occurs throughout subjective, social, and material dimensions:

"For me it's more like a game." To illustrate, he pointed to the games folder on his iPhone. "See, the app is right here, right next to Candy Crush." Erwin added: "It's so superficial. And as soon as you realize that and accept that, it starts to be fun." (pp. 1649-1650) 


\section{Preprint. \\ Full reference: Garda, M. B., \& Karhulahti, V. M. (2019). Let's Play Tinder! Aesthetics of a Dating App. Games and Culture, 1555412019891328. DOI: 10.1177/1555412019891328}

Erwin's phrasing "as soon as you realize that and accept that" implies that he has not always framed Tinder play as "gaming," and indeed, patterns of change and malleability emerge also in the other interviewees' voices. For instance, Sergio's engagement with the app started "just for fun [to] to pass the time [but] if I find someone I want to live with on Tinder it's ideal [but then again] not something that I'm really looking for" (pp. 1650). The paper contains further examples of this:

Reinout, 27, spoke about his use of Tinder with a self-deprecating humor. Like others, he had an ambitious streak when downloading the app that later faded: "At first I was really looking for a girlfriend. And now it's more like yeah, I just enjoy dating." He had learned how to play the game, but when asked to articulate his "ultimate goal," he returned to a relationship mindset: "The ultimate goal is to meet this one perfect match who will be my best friend for the rest of my life." (pp. 1650)

While the above examples evidence and explicate Tinder players' lively changes and development in the activity's framing, they also speak of its ambiguity or fuzziness. Regardless of whether one is playing the app to pass time, search for the one perfect match, or meet new friends, all these goals and motivations (among others) operate under a malleable system of contingent alacrity where active frames are constantly opportune to change (or overlapping accrual) and, thus, their function as "frames" becomes questionable.

A counterexemplary means for explaining the above is the ludic category of "transgressive play," presently understood as "play against the 'ideal' or 'implied' [by] innovation and, possibly, subversion, of finding, exploring and exploiting loop- holes in the game fabric" (Sunde'n, 2009, p. 2). Playing (with) Tinder can certainly be transgressive in multiple ways: hacking, political activism, and trolling all rep- resent known forms of engagement that likely fall out of the app's "ideal” use (e.g., Mason, 2016; March, Grieve, Marrington, \& Jonason, 2017; Richey, 2016). At the same time, however, there are no formal instructions or policies that would specify what a match in Tinder should mean and, thus, as long as the terms of service remain unviolated, transgressive or "wrong" kind of match seeking is a null concept.

In Tinder, the labels "nope" and "like" function as ludic symbols that have not been assigned any official signified meaning. Compared to the app's many compet- itors, the situation is distinct. In an almost identical "dating app," Bumble, the user must determine whether their aim when "liking" another user is to secure a date or if they are seeking new friendships. Moreover, the popular OkCupid asks the user to define their interest by the categories "long relationship," "short relationship," "hookup," and "friendship." Such specifications construct more or less explicit rules for framing the activity, which again makes breaking the rules (via frame violation) possible. By leaving its "nopes" and "likes" undefined, Tinder takes millions of diversely motivated individuals and allows them to conceptualize (and reconceptua- lize) their changing and flexible engagement without firm rules. While this hinders those interested in serious partner seeking (or other explicit goals), it also serves the app's economic ends by efficiently bringing a significant user base together with an implicit "promise" that there is something for everyone if they just keep looking hard enough.

The above aesthetic factors contribute to what philosopher Alexander Nehamas (2007) calls the "promise of happiness" element of human attraction. This element can be found in people's everyday charms, desires, and their subjective incomplete- ness, and while the "attraction of beauty always includes an erotic aspect, not every form of eroticism need manifest itself sexually" (p. 141). Attraction with the related charms and desires elicit reactions that are too complex to be thought of as mere pleasure, and in discovering them "we create it ourselves" (p. 132). The process of discovery and its (re)creation operates by the forward-looking promise of something exciting and unseen still awaiting in the associated entity.

The promise of happiness, as a phenomenological account of aesthetic engage- ment, opens a view to Tinder's diversely frameable mechanisms on multiple levels. Specifically, the core gameplay of swiping profile images sets out an intense chain of potential microscale desires and hopes that, swipe by swipe, add to the promise of happiness in something (whatsoever) becoming a conceivable possibility. Techni- cally, swiping "like" always 
leads to a potential match and, as such, produces positive feedback that keeps the player in the loop as long as they believe in the promise and perceive it with interest.

The design principles of Tinder support the players' promise production expli- citly. The fact that players are encouraged to reveal very little about themselves - contra earlier generations of dating software that pursue maximal information expo- sure (see Finkel, Eastwick, Karney, Reis, \& Sprecher, 2012; Timmermans \& Courtois 2018) - not only makes commencing play easy (not unlike casual mobile games) but also allows a wide range of interpretations that potentially contribute to the promises of happiness, as subjectively (re)created by individuals. The annul- ling multiplicity of frames constitutes an empowering alibi that validates engaging in romantic interactions via Tinder as play (in the nonserious and exploratory sense of the term) yet simultaneously, due to its abstraction of motives and scarcity of information, also feeds engagement that revitalizes malleable promise production.

\section{Conclusions}

Tinder's interpretive-kinesthetic gameplay constitutes a ludic platform that exceeds its labeled function of digital partner seeking. The design patterns that Tinder employs overlap with those of popular video games and slot machines, thereby inviting individuals to mechanical play where social interaction (and potential rela- tionships) serves as bets, currencies, and rewards.

On (game) mechanic and strategic levels, Tinder is based on profile construction and interpretation thereof. Within its game-specific cues and codes, Tinder players pursue and collect matches by means of communicative expression and interpreta- tion, the dimensions of which vary by culture, region, and time, among other factors.

In Tinder play, individuals frame the nature of their engagement subjectively. Regardless of (what players believe to be) their current underlying motivation, the app supports multiple overlapping frames that obscure the situation. As players gain access to the potentially recurring promise of happiness, the engagement occurs under the dual alibi of "play" and "romantic interaction," the concurrence of which enables their app-specific aesthetics.

\section{Funding}

The author(s) disclosed receipt of the following financial support for the research, authorship, and/or publication of this article: This article is funded by Academy of Finland projects "Sexuality and Play in Media Culture" (309382) and "Centre of Excellence in Game Culture Studies" (312396, 312397).

Note

1. Since September 2015, there has also been a third option called "Super Like." This feature allows the player to notify the target that they have been liked. Although the Super Like can break the app's mechanical binary, in practice, it does not due to its one per day limit. Players can buy more Super Likes, and thus the feature serves primarily the monetization of the free-to-play app. Its use(fullness) has been contested (see Thompson, 2017).

\section{References}

Arjoranta, J. (2015). Real-time hermeneutics: Meaning-making in ludonarrative digital games (PhD thesis). University of Jyva"skyla", Jyva“skylä, Finland.

Banks, J., Westerman, D. K., \& Sharabi, L. L. (2017). A mere holding effect: Haptic influ- ences on impression formation through mobile dating apps. Computers in Human Behavior, 76, 303-311. 
Bilton, N. (2014, October 29). Tinder, the fast-growing dating app, taps an age-old truth. The New York Times. Retrived from https://www.nytimes.com/2014/10/30/fashion/tinder-the- fast-growing-dating-app-taps-an-age-old-truth.html

Bogost, I. (2007). Persuasive games: The expressive power of videogames. MIT Press. Bullingham, L., \& Vasconcelos, A. C. (2013). 'The presentation of self in the online world': Goffman and the study of online identities. Journal of Information Science, 39, 101-112. Carpenter, C. J., \& McEwan, B. (2016). The players of micro-dating: Individual and gender differences in goal orientations toward micro-dating apps. First Monday, 21. doi:10.5210/

\section{fm.v21i5.6187}

Consalvo, M. (2009). There is no magic circle. Games and Culture, 4, 408-417.

Costikyan, G. (2013). Uncertainty in games. MIT Press.

David, G., \& Cambre, C. (2016). Screened intimacies: Tinder and the swipe logic. Social

Media p Society, 2. doi:10.1177/2056305116641976

Derk, I. K. (2016). Swipe left to detain: A procedural comparison between Tinder and papers,

please. Journal of Games Criticism, 3. Retrived from http://gamescriticism.org/articles/ derk-3-2

Duguay, S. (2017). Dressing up Tinderella: Interrogating authenticity claims on the mobile dating app Tinder. Information, Communication and Society, 20, 351-367.

Ermi, L, \& Mäyrä, F. (2005). Fundamental components of the gameplay experience: Analysing immersion. Worlds in Play: International Perspectives on Digital Games Research, 37, 37-53.

Finkel, E. J., Eastwick, P. W., Karney, B. R., Reis, H. T., \& Sprecher, S. (2012). Online dating: A critical analysis from the perspective of psychological science. Psychological Science in the Public Interest, 13, 3-66.

Fox, E. J. (2016, April 4). Here's how you can check if your partner is cheating on Tinder. Vanity Fair. Retrived from https://www.vanityfair.com/news/2016/04/check-tinder-chea ter-swipe-buster

Gatter, K., \& Hodkinson, K. (2016). On the differences between TinderTM versus online dating agencies: Questioning a myth. An exploratory study. Cogent Psychology, 3, doi:10.1080/ 23311908.2016.1162414

Goffman, E. (1956). The presentation of self in everyday life. University of Edinburgh Social Sciences Research Centre.

Goffman, E. (1986). Frame analysis. Northeastern University Press. (Original work published 1974) Hardey, M. (2008). The formation of social rules for digital interactions. Information,

Communication and Society, 11,1111-1131.

Järvinen, A. (2008). Games without frontiers: Theories and methods for game studies and

design (PhD dissertation). Tampere University Press, Tampere, Finland.

Karhulahti, V. (2011). Mechanic/aesthetic videogame genres: Adventure and adventure. In A. Lugmayr, H. Franssila, C. Safran, \& I. Hammouda (Eds.), Proceedings of the 15th

International Mindtrek Conference (pp. 71-74). ACM.

Karhulahti, V. (2015). Hermeneutics and Ludocriticism. Journal of Games Criticism, 2, 1-23. Kirkpatrick, G. (2015). The formation of gaming culture: UK gaming magazines, 1981-1995.

Springer.

Livingstone, C. (2005). Desire and the consumption of danger: Electronic gaming machines 
and the commodification of interiority. Addiction Research and Theory, 13, 523-534. March, E., Grieve, R., Marrington, J., \& Jonason, P. K. (2017). Trolling on Tinder $^{\circledR}$ (and other dating apps): Examining the role of the dark tetrad and impulsivity. Personality and

Individual Differences, 110, 139-143.

Mason, C. L. (2016). Tinder and humanitarian hook-ups: The erotics of social media racism.

Feminist Media Studies, 16, 822-837.

Nehamas, A. (2007). Only a promise of happiness: The place of beauty in a world of art.

Princeton University Press.

Orosz, G., To'th-Kira'ly, I., B\}othe, B., \& Melher, D. (2016). Too many swipes for today: The

development of the Problematic Tinder Use Scale (PTUS). Journal of Behavioral

Addictions, 5, 518-523.

Paasonen, S. (2018) Many Splendored Things: Thinking Sex and Play. Goldsmiths Press. Paul, M. (2018). Tinder business model - How does Tinder make money? Medium. Retrived

from https://medium.com/@monalisapaul_88268/tinder-business-model-how-does-tin der-make-money-498659f3f7cc

Perron, B., \& Arsenault, D. (2008). In the frame of the magic cycle: The circle(s) of gameplay. In B. Perron \& M. J. P. Wolf (Eds.), The video game theory reader 2 (pp. 131-154). Routledge.

Qiu, L., Lu, J., Yang, S., Qu, W., \& Zhu, T. (2015). What does your selfie say about you? Computers in Human Behavior, 52, 443-449.

Ranzini, G., \& Lutz, C. (2017). Love at first swipe? Explaining Tinder self-presentation and motives. Mobile Media and Communication, 5, 80-101.

Richey, L. A. (2016). Tinder humanitarians: The moral panic around representations of old relationships in new media. Javnost-The Public, 23, 398-414.

Schu"ll, N. D. (2012). Addiction by design: Machine gambling in Las Vegas. Princeton University Press.

Sevi, B., Aral, T., \& Eskenazi, T. (2018). Exploring the hook-up app: Low sexual disgust and high sociosexuality predict motivation to use Tinder for casual sex. Personality and Individual Differences, 133, 17-20.

Sicart, M. (2011). The ethics of computer games. MIT press.

Stenros, J. (2014). In defence of a magic circle: The social, mental and cultural boundaries of

play. Transactions of the Digital Games Research Association, 1, 147-185.

Summers, N. (2014). New Tinder security flaw exposed users' exact locations for months.

Bloomberg.

Sumter, S. R., Vandenbosch, L., \& Ligtenberg, L. (2017). Love me Tinder: Untangling

emerging adults' motivations for using the dating application Tinder. Telematics and

Informatics, 34, 67-78.

Sunde'n, J. (2009). Play as transgression: An ethnographic approach to queer game cultures. In 
DiGRA Conference Online Proceedings. DiGRA organization.

Thompson, R. (2017). Face it, super liking on Tinder is for losers. Mashable. Retrieved from

https://mashable.com/2017/02/09/super-like-tinder-losers/

Timmermans, E., \& Courtois, C. (2018). From swiping to casual sex and/or committed

relationships: Exploring the experiences of Tinder users. The Information Society, 34,

59-70.

Timmermans, E., \& De Caluwe', E. (2017a). Development and validation of the Tinder

Motives Scale (TMS). Computers in Human Behavior, 70, 341-350.

Timmermans, E., \& De Caluwe', E. (2017b). To Tinder or not to Tinder, that's the question: An individual differences perspective to Tinder use and motives. Personality and

Individual Differences, 110, 74-79.

Timmermans, E., De Caluwe', E., \& Alexopoulos, C. (2018). Why are you cheating on Tinder?

Exploring users' motives and (dark) personality traits. Computers in Human Behavior.

Advance online publication. doi:10.1016/j.chb.2018.07.040

Tyson, G., Perta, V. C., Haddadi, H., \& Seto, M. C. (2016, August 18-21). A first look at user

activity on Tinder. In Proceedings of the 2016 IEEE/ACM International Conference on

Advances in Social Networks Analysis and Mining (pp. 461-466). IEEE Press.

Vahlo, J. (2018). In gameplay: The invariant structures and varieties of the video game gameplay experience (PhD thesis). University of Turku, Turku.

Ward, J. (2017). What are you doing on Tinder? Impression management on a matchmaking mobile app. Information, Communication and Society, 20, 1644-1659.

Weiser, D. A., Niehuis, S., Flora, J., Punyanunt-Carter, N. M., Arias, V. S., \& Baird, R. H. (2018). Swiping right: Sociosexuality, intentions to engage in infidelity, and infidelity experiences on Tinder. Personality and Individual Differences, 133, 29-33. 